\title{
Research on Psychological Teaching based on the Background of Epidemic Prevention and Control in COVID-19
}

\author{
Mengting $\mathrm{Xi}$ \\ Yichun Vocational Technology College, Yichun 336000, China \\ 115189142@qq.com
}

\begin{abstract}
After the occurrence of new pneumonia, in order to avoid people's serious psychological problems under sudden crisis, the relevant departments of our country have formulated a series of intervention guiding principles, aiming at avoiding the occurrence of psychological problems through timely psychological counseling. In view of the students in higher vocational colleges, psychological intervention should be carried out as early as possible by mastering the students' psychological dynamics, to minimize the psychological impact on students caused by the epidemic situation.
\end{abstract}

Keywords: Psychological Teaching; COVID-19 Epidemic.

\section{Introduction}

The outbreak of COVID-19 not only threatens the lives of thousands of people, but also brings some panic and anxiety to people's psychology. What is the main anxiety of students facing COVID19? In order to fully understand students' psychological status and solve students' psychological problems in a targeted manner, higher vocational colleges recently conducted questionnaire survey activities on campus WeChat official account and official website, aiming at the problems existing in students' psychology, study and life during the COVID-19 epidemic, so as to fully grasp the current psychological status of students in higher vocational colleges.

\section{Psychological Representation under the Influence of Epidemic Situation}

\subsection{Credulity of False Information}

Because of the sudden, gathering and spreading characteristics of the epidemic, teenagers are faced with a large amount of information in a short time, and they are prone to blindly follow and believe in false information. For example, rumors such as "smoking and drinking can prevent novel coronavirus infection", "Chinese medicine formula for preventing pneumonia", "all infected people are old people, and children are fine" and "taking antibiotics and Shuanghuanglian can prevent novel coronavirus" have been circulated on the Internet, which have a certain impact on teenagers' psychology. A survey involving 16,352 students showed that $1.2 \% \sim 35.6 \%$ of the students believed the false information about the epidemic. It can be seen that teenagers are in the stage of intense curiosity, and have a strong thirst for knowledge about the prevention and treatment of epidemic situation. However, some teenagers have not formed the literacy of correctly collecting and processing information by means of information technology, and lack scientific analysis and interpretation of epidemic situation-related information, and adopt a credulous attitude towards complicated information.

\subsection{Panic about the Epidemic}

COVID-19 is highly contagious, dangerous and has a wide range of influences, which makes teenagers panic about the epidemic situation. Most teenagers implement personal protective measures in a targeted manner, actively cooperate with disinfection at home and work at home required by schools, and actively wear masks to reduce or avoid contact with the outside world. However, some groups have irrational panic and avoidance psychology. A survey on the public's cognition, attitude and behavior in the face of the epidemic shows that $96.31 \%$ of the people are in an excessive state of fear, tension and worry, and some of them think that the epidemic will have a serious impact. [2] This 
Volume 3 (2021)

kind of excessive tension on the epidemic situation will cause teenagers to be influenced by the public opinion of relatives and friends around them, and aggravate the psychological state of teenagers falling into panic. When the epidemic situation spreads rapidly, this state of mind will gradually increase. The education departments in many areas held meetings and said that schools of all levels and types can only start school after the epidemic situation has been effectively controlled. On the one hand, it ensures the health of teachers and students in schools; on the other hand, it is hoped that through the control of the epidemic situation and the improvement of the treatment effect, the panic of young students can be reduced, so that they can return to normal study and life after the school starts and resumes.

\subsection{Long-term Loneliness at Home}

Long-term home isolation has a certain impact on teenagers' mental health. Some teenagers are prone to negative situations such as language barrier and bad mood due to lack of face-to-face communication, which will seriously affect teenagers' body resistance and induce physical and mental diseases. According to the home isolation during the COVID-19 epidemic, some studies have shown that staying at home for a long time has a great influence on emotions, among which $23.6 \%$ of the groups feel anxious in the process of staying at home, $28.9 \%$ of the groups have mild depression tendency, and $7.7 \%$ of the groups reach moderate, severe and extremely severe depression, among which the number of students is as high as $6.7 \%$. Being at home for a long time makes active teenagers lack direct contact with the outside world, and loneliness will bring them greater psychological pressure, and even induce serious psychological problems such as anxiety and depression.

\section{Causes of Adolescent Negative Psychology}

\subsection{Abuse of Mobile Terminals and Social Applications}

The isolation at home during the epidemic period provided teenagers with the opportunity to use the network, and mobile terminals with high convenience gradually became the most frequently used devices in teenagers' home study. On the one hand, social applications have contributed to the spread of rumors in this epidemic, and teenagers can easily find and obtain various kinds of information from them. The strong network of social applications has further intensified the fission spread of rumors and provided a hotbed for the spread of false information. At the same time, since the arrival of the media era, the spread frequency of epidemic rumors has increased, and the logical thinking of teenagers has not been completely perfected, so it is difficult to extract the true content from various complicated information. On the other hand, stimulated by the use of high-frequency mobile devices, teenagers' online behavior is prone to get out of control of impulse, and shows the damage of individual psychological function caused by abusing the Internet, which may even increase the possibility of teenagers suffering from Internet addiction and seriously hinder the physical and mental development of teenagers.

\subsection{Lack of School and Family-related Education}

The outbreak has sudden characteristics, so it is difficult for schools and families to complete the counseling of adolescents' psychological problems in a short time. Schools and families, as natural educational partners, need to create a home-school joint atmosphere to resist the epidemic "psychological defense" and enhance the psychological "immunity" of teenagers. However, at present, schools and families do not know enough about the epidemic prevention and control, so it is difficult to complete the introduction and popularization of the epidemic at the first time, and they also lack the prevention and education of adolescents' psychological problems, showing a state of insufficient mental health education. On the other hand, when the COVID-19 epidemic broke out, the school teachers and family members would actively carry out the epidemic prevention and control work, which to a certain extent reduced their attention to the psychological changes of teenagers, and their psychological counseling could not be carried out effectively and in time. Teenagers have special 
Volume 3 (2021)

psychological characteristics-imaginary audience and personal fable. When faced with sudden epidemic, they can easily overestimate their ability to solve psychological problems and lack initiative in seeking help from teachers and parents.

\subsection{Limitations of the Law of Teenagers' Physical and Mental Development}

Selye, a Canadian scholar, put forward the stress response theory in 1937, which proposed that when the human body is stimulated by various stressors (external, internal and social), it will cause individual nonspecific response. At present, the COVID-19 epidemic is a social stimulus factor, and its influence on the organism includes physiological reaction and psychological reaction. The physiological responses were sympathetic nerve excitement, increased secretion of pituitary and adrenal cortex hormones, increased blood sugar, blood pressure, heart rate and respiration. Psychological reaction includes emotional reaction, self-defense reaction and coping reaction. The most common emotions are anxiety, anger, guilt, fear, depression, learned helplessness and so on. The result of stress response is not only caused by stimuli, but also related to the individual's understanding of stressors and the individual's experience in dealing with stress events.

Students in five-year higher vocational colleges are generally aged from 145 to 178, which coincides with adolescence or early youth. From a psychological point of view, this age belongs to the early youth, which is the most rapid, vigorous and critical period for students' physical and mental development. Due to the fierce psychological changes, this period is also a period in which people are prone to psychological confusion and psychological conflicts. The current COVID-19 epidemic is the most concerned issue in China. People's understanding of the epidemic mainly comes from the Internet. Adults with mature minds will inevitably be greatly affected, not to mention that these immature students, combined with the change of lifestyle, originally can move around, but during the epidemic, they are confined in a relatively narrow space, with nowhere to move and nowhere to release pressure, which is easy to cause trouble to their emotions, resulting in increased anxiety, tension and depression (depression); Cognitive ability is reduced; Self-concept is not clear.

\section{Counseling Strategies for Students' Psychological Status in COVID-19}

\subsection{Carry out Health Education to Ensure the Prevention and Control of Teenagers' Psychology}

Facing the epidemic situation, it is normal for people to have anxiety, panic, worry and fear. During the epidemic, students feel uncertain about the epidemic, and if all departments can formulate guiding principles in time and guide them so that students can get rid of bad emotions as soon as possible, then the epidemic is a psychological experience for students and plays a positive role in promoting their future growth. On the contrary, the influence of Long-term negative emotions will lead to changes in students' psychological physiology and have adverse effects on students.

It is self-evident that the importance of health education activities to ensure the prevention and control of adolescents' psychological problems in the epidemic situation is obvious, which should be regarded as an effective starting point for prevention and control of adolescents' psychological problems. At present, China has achieved good results in psychological assistance and health education for confirmed patients. For example, health education activities such as moderate exercise and art competition between doctors and patients have appeared in Wuhan Fangcang Hospital, which effectively relieve the pressure of confirmed patients, enhance their confidence and help them recover as soon as possible. Psychological prevention and control for teenagers can also be done through health education, so that teenagers can have beneficial and simple activities together with their families in the process of staying at home, which can not only enhance teenagers' understanding of epidemic prevention and control, but also enable them to form a positive and optimistic attitude and reduce the occurrence of psychological problems among teenagers. Schools should also continue to strengthen the education of popular science for adolescents, show students the latest public 
information, and publish a list of common rumors, to reduce the frequency of rumors spreading within adolescents and reduce their fear of the epidemic.

During the epidemic period, schools can make full use of new media platforms such as official website and WeChat official account to push prevention and control related knowledge and psychological counseling articles to all students every day, and provide students with psychological health care knowledge from five aspects: understanding the nature of pneumonia infected in novel coronavirus, taking active and effective preventive measures, actively seeking psychological support through more exchanges with others, discovering the positive significance of life, and seeking help from professional mental health personnel. By providing psychological assistance hotline service, students in need are wholeheartedly helped to reduce the psychological interference of the epidemic. Inform the current situation of prevention and control and the deployment of school prevention and control in time, so that students can know, understand and cooperate with the epidemic prevention and control work, face the epidemic with a positive and optimistic attitude, and overcome the epidemic with tenacious will and firm belief. At the same time, in view of the long vacation time of students, various colorful home activities can be carried out, such as home sports display, manual works display, housework display, etc., which are sent to the campus network by recording small videos for everyone to enjoy, and the outstanding works are evaluated and rewarded, so that students can turn their attention to more meaningful things in their lives and eliminate the negative impact of the epidemic.

\subsection{Strengthen the Linkage between Home and School and Build a Multi-subject Education System}

It is also an important way of mental health education to strengthen the linkage between home and school and form the synergy between home and school. Teachers and parents should actively communicate and exchange students' learning and thinking, to protect teenagers' healthy home. On the one hand, teachers should clearly express the importance of mental health education, guide parents to attach importance to teenagers' mental state, and reasonably formulate guiding opinions on prevention and control of teenagers' psychological problems, while parents should actively complete communication with schools, enhance their understanding and cooperation on school epidemic prevention and online education, and make concerted efforts with schools to ensure teenagers' mental health. On the other hand, it is necessary to strengthen the skills education of youth epidemic prevention and control, so that teenagers can further develop the living habit of preventing COVID19 and master the life skills of preventing COVID-19 based on understanding the theoretical knowledge, such as reducing group gathering activities, learning to wear and disinfect common medical masks, and learning the "seven-step washing technique" to wash their hands. This long stay at home also provides excellent life education opportunities for schools and families. Teachers and parents should actively guide young people to get help in the event of public crisis through various means, and know how to seek help.

\section{Conclusion}

To sum up, at present, the research on prevention and control of adolescents' psychological state changes and psychological problems in the epidemic situation is slightly insufficient, but adolescents are negatively affected by the COVID-19 epidemic situation, so it is urgent to establish a targeted psychological counseling system. Therefore, while carrying out epidemic prevention and control in a timely and effective manner, all sectors of society should also pay more attention to the mental state of young people, actively carry out mental health education and health education activities, strengthen the rational and scientific linkage mechanism between schools and families, always pay attention to the psychological changes of students at home, and make good use of the online education platform to broaden diversified channels of counseling, so as to lay a solid foundation for young students to live and start school normally. 


\section{References}

[1] Cai Meng-sheng, Tang Qiang. Research on the police's psychological cognition and law enforcement behavior under the COVID-19 epidemic-based on the statistical analysis of 1075 questionnaires nationwide [J]. Journal of Jiangsu Police Academy, 2021(2020-5):74-81.

[2] Luo Jiayi, Li Huahua, Mai Baoxin. Investigation and analysis of mental health status of middle school students returning to school under the background of COVID-19 epidemic [J]. Mental Health Education in Primary and Secondary Schools, 2020(22):12-15.

[3] Lu Yongping, Zhang Qin, Yang Ping, et al. Investigation and analysis of online teaching of Peking Union Medical College during the prevention and control of COVID-19 epidemic [J]. Medical Teaching Research in Colleges and Universities (Electronic Edition), Vol. 10, No.3, 2020, pp. 8-14, 2020.

[4] Ding Minjiang, Hu Chunfu. Study on the influence of COVID-19 epidemic on college students' psychological behavior [J]. Journal of Huaihai Institute of Technology (Social Science Edition), 2020, 018 (002): 129-140.

[5] Cao Jijuan, Guo Jianjin, Liu Yingtong. Study on the influencing factors of college students' use of intelligent teaching system under the background of COVID-19 epidemic [J]. Science and Technology Economic Guide, 2020, v.28; No.715(17):110-112.

[6] Lu Qingyan. Psychological characteristics and coping strategies of vocational college students under the normalization of epidemic prevention and control in COVID-19 [J]. Educational Research, 2021, 4(6):5556. 